PROCEEDINGS OF THE

AMERICAN MATHEMATICAL SOCIETY

Volume 127, Number 5, Pages 1257-1263

S 0002-9939(99)04622-5

Article electronically published on January 27, 1999

\title{
ON THE REDUCTION NUMBER OF SOME GRADED ALGEBRAS
}

\author{
HENRIK BRESINSKY AND LÊ TUÂN HOA
}

(Communicated by Wolmer V. Vasconcelos)

\begin{abstract}
The main result of the paper confirms, for generic coordinates, a conjecture which states that $r(R / I) \leq r(R / i n(I))$. Here $I$ is a homogeneous polynomial ideal in $R$ and $r(R / I)$ and $r(R /$ in $(I))$ are the reduction numbers.
\end{abstract}

\section{INTRODUCTION}

Let $R=k\left[x_{1}, \ldots, x_{n}\right]$ be a polynomial ring, $k$ an infinite field and $I \neq 0$ a homogeneous ideal in $R$. We set $\mathfrak{m}=\left(x_{1}, \ldots, x_{n}\right) R / I$.

Definition 1. A homogeneous ideal $J \subseteq \mathfrak{m}$ is called a reduction of $\mathfrak{m}$ if $\mathfrak{m}^{r+1}=J \mathfrak{m}^{r}$ for some integer $r \geq 0$. $J$ is called a minimal reduction, if $J$ does not properly contain a reduction of $\mathfrak{m}$ (see $[6]$ ). The reduction number of $\mathfrak{m}$ with respect to a minimal reduction $J$ of $\mathfrak{m}$, denoted by $r_{J}(\mathfrak{m})$ or $r_{J}(R / I)$, is the smallest $r \geq 0$ such that $\mathfrak{m}^{r+1}=J \mathfrak{m}^{r}$. The (absolute) reduction number of $\mathfrak{m}$, denoted by $r(\mathfrak{m})$ or $r(R / I)$, is the infimum of $r_{J}(\mathfrak{m})$ over all possible minimal reductions $J$ of $\mathfrak{m}$. (This notion was introduced by J. Sally and has proven to be an important invariant (see, e.g. [7], [8]).)

Let in $(I)$ be the initial ideal of $I$ with respect to some admissible term order on the terms of $R$ (see [4], Chapter 15, or [3]). $i n(I)$ is a monomial ideal and frequently contains important information about $I$ (see [4], Section 15.10, and [8]). A natural question therefore is to ask what relationship exists between $r(R / I)$ and $r(R / \operatorname{in}(I))$. In [8] it was conjectured that

$$
r(R / I) \leq r(R / \operatorname{in}(I))
$$

The purpose of this note is to prove that the conjecture is true for generic coordinates (Theorem 12). This supports the general conjecture to the extent that in all examples done by us $r(R / \operatorname{gin}(I))$ is minimal among $r(R / i n(I))$, where $\operatorname{gin}(I)$ is the initial ideal of $I$ in generic coordinates (for a definition of $\operatorname{gin}(I)$ see [4], Section 15.9). We obtain our result as a consequence of our study of the reduction number of Borel-fixed ideals in Section 3. Section 2 gives some preliminary results on the reduction number of a special class of monomials ideals. In conclusion in Section

Received by the editors April 18, 1997 and, in revised form, August 6, 1997.

1991 Mathematics Subject Classification. Primary 13C05, 13 A15.

Key words and phrases. Monomial ideal, Borel-fixed ideal, generic coordinates, reduction number. 
4, we make a remark on the relationship between the reduction number and the maximal degree in a minimal homogeneous generating set of $I$.

\section{Preliminary RESUlts}

We assume throughout that $R, I$ and $\mathfrak{m}$ are defined as in Section 1 and $\operatorname{dim}(R / I)$ $=d$. By $\bar{y}$ we denote the image of $y \in R$ in $R / I$.

Definition 2. If $M$ is any $\mathbf{Z}$-graded $R$-module, we define

$$
a(M)= \begin{cases}\max \left\{p ; M_{p} \neq 0\right\} & \text { if } M \neq 0, \\ -\infty & \text { if } M=0 .\end{cases}
$$

By [6], a minimal reduction of $\mathfrak{m}$ can always be generated by a system of parameters. The following lemma is well known. We provide a proof for the convenience of the reader.

Lemma 3. The ideal $J=\left(\bar{y}_{1}, \ldots, \bar{y}_{d}\right) \subseteq R / I$ is a minimal reduction of $\mathfrak{m}$ iff $\bar{y}_{1}, \ldots, \bar{y}_{d}$ is a system of parameters (s.o.p.) of $R / I$ with $\bar{y}_{i}$ linear forms, $1 \leq i \leq d$. In this case

$$
r_{J}(\mathfrak{m})=a\left(R /\left(I, y_{1}, \ldots, y_{d}\right)\right) .
$$

Proof. Let $r=r_{J}(\mathfrak{m})$ and $a=a\left(R /\left(I, y_{1}, \ldots, y_{d}\right)\right)$. We have $\mathfrak{m}^{p+1}=\left(\bar{y}_{1}, \ldots, \bar{y}_{d}\right) \mathfrak{m}^{p}$ for all $p \geq r$. Suppose some element, say $\bar{y}_{d}$, has degree greater than 1 . Then $[R / I]_{p+1} \subseteq \bar{y}_{1}[R / I]_{p}+\cdots+\bar{y}_{d-1}[R / I]_{p}$, where $[R / I]_{i}$ are the homogeneous elements of degree $i$ in $R / I$. From this it follows that $\mathfrak{m}^{p+1} \subseteq\left(\bar{y}_{1}, \ldots, \bar{y}_{d-1}\right)$, a contradiction since $\operatorname{dim}(R / I)=d$. Also $[R / I]_{p+1} \subseteq\left(\bar{y}_{1}, \ldots, \bar{y}_{d}\right)$, hence $R_{p+1} \subseteq\left(I, y_{1}, \ldots, y_{d}\right)$, and thus $a \leq r$. Conversely if $\bar{y} \in \mathfrak{m}^{a+1}$, then its image $\overline{\bar{y}} \in R /\left(I, y_{1}, \ldots, y_{d}\right)$ is 0 , since otherwise it would have degree at least $a+1$, which is impossible. This means $\bar{y} \in\left(\bar{y}_{1}, \ldots, \bar{y}_{d}\right)$, i.e. $\bar{y}=\bar{y}_{i_{1}} \bar{z}_{1}+\cdots+\bar{y}_{i_{q}} \bar{z}_{q}$, where $\bar{z}_{j} \in R / I$ and $1 \leq i_{1}<\cdots<i_{q} \leq d$. W.l.o.g. we may assume $q$ to be minimal. Then, comparing degrees on both sides, we have $\operatorname{deg}\left(\bar{z}_{j}\right) \geq \operatorname{deg}(\bar{y})-\operatorname{deg}\left(\bar{y}_{i_{j}}\right)=a$, i.e. $\bar{z}_{j} \in \mathfrak{m}^{a}$. Thus $\mathfrak{m}^{a+1} \subseteq\left(\bar{y}_{1}, \ldots, \bar{y}_{d}\right) \mathfrak{m}^{a}$ which implies $\mathfrak{m}^{a+1}=\left(\bar{y}_{1}, \ldots, \bar{y}_{d}\right) \mathfrak{m}^{a}$; hence $a \geq r$.

Proposition 4. $r(R / I) \geq \min \{\operatorname{deg}(F) ; F \in I, F$ homogeneous $\}-1$.

Proof. Let $J=\left(\bar{y}_{1}, \ldots, \bar{y}_{d}\right)$ be a minimal reduction of $\mathfrak{m}$. Since $I \neq 0, I \nsubseteq\left(y_{1}, \ldots, y_{d}\right)$. Set $I^{\prime}:=\left(I, y_{1}, \ldots, y_{d}\right) /\left(y_{1}, \ldots, y_{d}\right) \subseteq R^{\prime}: \cong R /\left(y_{1}, \ldots, y_{d}\right)$. Then

$$
\begin{aligned}
r_{J}(R / I) & =a\left(R /\left(I, y_{1}, \ldots, y_{d}\right)\right)=a\left(R^{\prime} / I^{\prime}\right) \\
& \geq \min \left\{\operatorname{deg}\left(F^{\prime}\right) ; F^{\prime} \in I^{\prime}, F^{\prime} \text { homogeneous }\right\}-1 \\
& \geq \min \{\operatorname{deg}(F) ; F \in I, F \text { homogeneous }\}-1 .
\end{aligned}
$$

We note that this lower bound can actually be attained (see Example 7). The following result will be useful for the study of reduction numbers of a certain class of monomial ideals.

Lemma 5. Assume that $I$ is a monomial ideal of $R$ such that $\bar{x}_{n-d+1}, \ldots, \bar{x}_{n}$ is a s.o.p. of $R / I$. Then any minimal reduction $J$ of $\mathfrak{m}$ is generated by $d$ linear forms $\bar{y}_{1}, \ldots, \bar{y}_{d}$ with

$$
y_{i}=x_{n-d+i}+a_{i, 1} x_{1}+\cdots+a_{i, n-d} x_{n-d}, 1 \leq i \leq d,
$$

where $a_{i, j} \in k, 1 \leq j \leq n-d$. 
Proof. Let $\mathfrak{p}$ be a highest dimensional associated prime ideal of $I$. Then $x_{n-d+1}, \ldots$, $x_{n}$ are not in $\mathfrak{p}$. Since any associated prime ideal of a monomial ideal is generated by a subset of variables and $\operatorname{dim}(R / \mathfrak{p})=d$, it follows that $\mathfrak{p}=\left(x_{1}, \ldots, x_{n-d}\right)$. Let $S=R / I$ and for an integer $i, 1 \leq i \leq d$, let $\mathfrak{n}_{i}:=\left(x_{1}, \ldots, x_{n-d}, x_{n-d+i+1}, \ldots, x_{n}\right) S$. By Lemma 3 we may assume that $y_{1}, \ldots, y_{d}$ are linear forms. If there is no $i, 1 \leq i \leq$ $d$, such that a nonzero summand of $y_{i}$ is a multiple of $x_{n-d+1}$, then $\left(\bar{y}_{1}, \ldots, \bar{y}_{d}\right) \subseteq \mathfrak{n}_{1}$; thus $\operatorname{dim}\left(S /\left(\bar{y}_{1}, \ldots, \bar{y}_{d}\right)\right) \geq 1$, a contradiction. Assume w.l.o.g.

$$
y_{1}=x_{n-d+1}+\sum_{j=1, j \neq n-d+1}^{n} b_{1 j} x_{j} .
$$

Subtracting multiples of $y_{1}$ if need be, we eliminate $x_{n-d+1}$ from $y_{i}, i \neq 1$. Suppose there is no $i, 2 \leq i \leq d$, such that a nonzero summand of $y_{i}$ is a multiple of $x_{n-d+2}$. Since then $\left(\bar{y}_{2}, \ldots, \bar{y}_{d}\right) \subseteq \mathfrak{n}_{2}, \operatorname{dim}\left(S /\left(\bar{y}_{2}, \ldots, \bar{y}_{d}\right)\right) \geq \operatorname{dim}\left(S / \mathfrak{n}_{2}\right)=2$, a contradiction. W.l.o.g. assume

$$
y_{2}=x_{n-d+2}+\sum_{j=1}^{n-d} b_{2 j} x_{j}+\sum_{j=n-d+3}^{n} b_{2 j} x_{j} .
$$

Subtracting multiples of $y_{2}$ if need be, we eliminate $x_{n-d+2}$ from $y_{i}, i \neq 2$. Continuing, we obtain a s.o.p. $y_{i}, 1 \leq i \leq d$, as claimed.

Corollary 6. Assume that $I$ is a monomial ideal as in Lemma 5. Then for any minimal reduction $J$ of $\mathfrak{m}$ we have

$$
r_{J}(\mathfrak{m}) \leq r_{\left(x_{n-d+1}, \ldots, x_{n}\right)}(\mathfrak{m}) .
$$

Proof. By Lemma 5 we may assume that $J=\left(\bar{y}_{1}, \ldots, \bar{y}_{d}\right)$, where $y_{i}=x_{n-d+i}+$ $a_{i, 1} x_{1}+\cdots+a_{i, n-d} x_{n-d}, 1 \leq i \leq d$. Consider $k\left[x_{1}, \ldots, x_{n-d}\right]$ as a subring of $R$ and set $I_{1}=I \cap k\left[x_{1}, \ldots, x_{n-d}\right], I_{2}=\left(I, y_{1}, \ldots, y_{d}\right) \cap k\left[x_{1}, \ldots, x_{n-d}\right]$. Then $R /\left(I, x_{n-d+1}, \ldots, x_{n}\right) \cong k\left[x_{1}, \ldots, x_{n-d}\right] / I_{1}, R /\left(I, y_{1}, \ldots, y_{d}\right) \cong k\left[x_{1}, \ldots, x_{n-d}\right] / I_{2}$ and $I_{1} \subseteq I_{2}$. Therefore the corollary now follows by Lemma 3 .

The following examples show that the difference between $r_{\left(x_{n-d+1}, \ldots, x_{n}\right)}(\mathfrak{m})$ and $r(\mathfrak{m})$ in the setting of Lemma 5 can be very large. They also show that the reduction number $r_{J}(\mathfrak{m})$ with respect to a minimal reduction $J$ of $\mathfrak{m}$ depends very much on the choice of $J$. Together with Corollary 6 this explains why it is still difficult to calculate $r(R / I)$, even for monomial ideals satisfying Lemma 5 .

Example 7. Let $a_{1}>a_{2}>\cdots>a_{n} \geq 2$ be positive integers.

1. The ideal

$$
I=\left(x_{1}^{a_{1}}, \ldots, x_{n-1}^{a_{n-1}}, x_{i} x_{j}, 1 \leq i<j \leq n\right)
$$

is a one-dimensional ideal and $\left(x_{n}\right) R / I$ is a parameter ideal of $R / I$. By Lemma 3 we have

$$
r_{J}(\mathfrak{m})= \begin{cases}a_{1}-1 & \text { if } J=\left(x_{n}\right) R / I \\ a_{i+1}-1 & \text { if } J=\left(x_{n}-x_{1}-\cdots-x_{i}\right) R / I, 1 \leq i<n-1, \\ 1 & \text { if } J=\left(x_{n}-x_{1}-\cdots-x_{n-1}\right) R / I\end{cases}
$$

Hence $r(\mathfrak{m})=1$.

2. The ideal

$$
I=\left(x_{1}^{a_{1}}, x_{1} x_{2}^{a_{2}}, \ldots, x_{1} x_{n}^{a_{n}}\right)
$$


is a one-codimensional ideal and $\left(x_{2}, \ldots, x_{n}\right) R / I$ is a parameter ideal of $R / I$. Again by Lemma 3 we get

$$
r_{J}(\mathfrak{m})= \begin{cases}a_{1}-1 & \text { if } J=\left(x_{2}, \ldots, x_{n}\right), \\ a_{i} & \text { if } J=\left(x_{2}, \ldots, x_{i-1}, x_{i}-x_{1}, x_{i+1}, \ldots, x_{n}\right), 2 \leq i \leq n .\end{cases}
$$

Since $a_{n}+1$ is the least degree in the above generating set, by Proposition 4 , $r(\mathfrak{m})=a_{n}$.

\section{BOREL-FIXED IDEALS}

In this section we study the reduction number of so called Borel-fixed ideals.

Definition 8. The action of a matrix $g=\left(g_{i j}\right) \in G L(n, k)$ on $k\left[x_{1}, \ldots, x_{n}\right]$ is defined by $g\left(x_{i}\right)=\sum_{j=1}^{n} g_{j i} x_{j}$. Let $B=\left\{g ; g \in G L(n, k), g_{i j}=0\right.$ for $\left.j<i\right\}$ be the Borel subgroup of $G L(n, k)$. An ideal $I$ of $R$ is called Borel fixed if $g(I)=I$ for all $g \in B$.

Definition 9. A monomial ideal $I$ is said to be stable if $m \in I$ and $x_{i} \mid m$ imply $m x_{j} / x_{i} \in I$ for all $j \leq i$.

It follows immediately that a stable ideal $I$ is Borel-fixed. If $\operatorname{char}(k)=0$, then by [3], Proposition 2.7, Borel-fixed ideals are stable ideals. Borel-fixed ideals are important, because for any term order such that $x_{1}>x_{2}>\cdots>x_{n}$, in generic coordinates the initial ideal, $\operatorname{gin}(I)$, is Borel-fixed.

Lemma 10. Let I be a d-dimensional Borel-fixed monomial ideal. Then:

(i) $\left(x_{n-d+1}, \ldots, x_{n}\right) R / I$ is a parameter ideal of $R / I$ and $x_{n-d}^{\alpha}$ is a part of the minimal monomial generating set of $I$ for some $\alpha>0$.

(ii) If I is stable, then $r_{\left(x_{n-d+1}, \ldots, x_{n}\right)}(\mathfrak{m})=\alpha-1$.

Proof. (i) See [4], Corollary 15.25.

(ii) Let $J=\left(x_{n-d+1}, \ldots, x_{n}\right)$. By (i) $x_{n-d}^{\alpha}$ is part of a minimal monomial generating set of $I$ for some $\alpha>0$. Hence by Lemma $3, r_{J}(\mathfrak{m}) \geq \alpha-1$. Assume $r:=$ $r_{J}(\mathfrak{m}) \geq \alpha$. Again by Lemma 3 and (i), there is a monomial $m=x_{1}^{a_{1}} \ldots x_{n-d}^{a_{n-d}} \notin I$ such that $\operatorname{deg}(m)=r$. Since $I$ is stable, this implies that $x_{n-d}^{r} \notin I$, a contradiction. Thus $r=\alpha-1$.

Without the assumption that $I$ is a stable ideal, Lemma 10 (ii) is false. Let $\operatorname{char}(k)=p>0$. Then $I=\left(x_{1}^{p}, x_{2}^{p}, x_{1} x_{3}^{p}\right) \subset k\left[x_{1}, x_{2}, x_{3}\right]$ is Borel-fixed, but $r_{\left(x_{3}\right)}(R / I)=2 p-2$.

In contrast to Example 7, the following result shows that the reduction numbers of Borel-fixed ideals behave well.

Theorem 11. Assume that $I$ is a Borel-fixed monomial ideal. Then for an arbitrary minimal reduction $J$ of $R / I$ we have $r(R / I)=r_{J}(R / I)$. Thus the reduction number $r(R / I)$ does not depend on the choice of a minimal reduction.

Moreover, if the ideal is stable, then $r(R / I)=\alpha-1, \alpha$ as in Lemma 10.

Proof. By Lemma 10 it suffices to show that $r_{J}(\mathfrak{m})=r_{\left(x_{n-d+1}, \ldots, x_{n}\right)}(\mathfrak{m})$. By Lemma 5 we may assume that $J=\left(\bar{y}_{1}, \ldots, \bar{y}_{d}\right)$, where

$$
y_{i}=x_{n-d+i}+a_{i, 1} x_{1}+\cdots+a_{i, n-d} x_{n-d}, \quad 1 \leq i \leq d .
$$

Let $I_{1}$ be the ideal in $k\left[x_{1}, \ldots, x_{n-d}\right]$, which is obtained from $I$ by replacing the variable $x_{n-d+i}$ in each monomial $m \in I$ by $-\left(a_{i, 1} x_{1}+\cdots+a_{i, n-d} x_{n-d}\right), 1 \leq i \leq d$. 
Then we have $R /\left(I, y_{1}, \ldots, y_{d}\right) \cong k\left[x_{1}, \ldots, x_{n-d}\right] / I_{1}$. Also $R /\left(I, x_{n-d+1}, \ldots, x_{n}\right) \cong$ $k\left[x_{1}, \ldots, x_{n-d}\right] / I_{2}$, where $I_{2}=I \cap k\left[x_{1}, \ldots, x_{n-d}\right]$. Clearly $I_{2} \subseteq I_{1}$. Now let $m=$ $x_{1}^{b_{1}} \ldots x_{n}^{b_{n}} \in I$. Set

$$
g=\left(\begin{array}{ccccccc}
1 & 0 & \cdots & 0 & -a_{1,1} & \cdots & -a_{d, 1} \\
0 & 1 & \cdots & 0 & -a_{1,2} & \cdots & -a_{d, 2} \\
\cdot & \cdot & \cdots & \cdot & \cdot & \cdots & \cdot \\
0 & 0 & \cdots & 1 & -a_{1, n-d} & \cdots & -a_{d, n-d} \\
0 & 0 & \cdots & 0 & 1 & \cdots & 0 \\
\cdot & \cdot & \cdots & \cdot & \cdot & \cdots & \cdot \\
0 & 0 & \cdots & 0 & 0 & \cdots & 1
\end{array}\right) .
$$

Since $I$ is Borel-fixed,

$$
g(m)=x_{1}^{b_{1}} \ldots x_{n-d}^{b_{n-d}} \prod_{i=1}^{d}\left[x_{n-d+i}-\left(a_{i, 1} x_{1}+\cdots+a_{i, n-d} x_{n-d}\right)\right]^{b_{n-d+i}} \in I .
$$

Note that if $f+g$ belongs to a monomial ideal $I$, then both polynomials $f$ and $g$ belong to $I$ too. Hence the above relation implies that

$$
x_{1}^{b_{1}} \ldots x_{n-d}^{b_{n-d}} \prod_{i=1}^{d}\left[-\left(a_{i, 1} x_{1}+\cdots+a_{i, n-d} x_{n-d}\right)\right]^{b_{n-d+i}} \in I .
$$

This means $I_{1} \subseteq I_{2}$, which yields $I_{1}=I_{2}$. Thus by Lemma 3 we get $r_{J}(\mathfrak{m})=$ $r_{\left(x_{n-d+1}, \ldots, x_{n}\right)}(\mathfrak{m})$.

Now we can state and prove the main result of this paper, which gives partially a positive answer to the conjecture of Vasconcelos mentioned in the introduction.

Theorem 12. Assume that in $(I)$ is a Borel-fixed ideal. Then $r(R / I) \leq r(R /$ in $(I))$.

In particular in generic coordinates the above inequality holds for any admissible term order.

Proof. By Lemma 10, $\bar{x}_{n-d+1}, \ldots, \bar{x}_{n}$ is a s.o.p. of $\left.R / i n(I)\right)$. Since

$$
\left(i n(I), x_{n-d+1}, \ldots, x_{n}\right) \subseteq i n\left(I, x_{n-d+1}, \ldots, x_{n}\right),
$$

$\bar{x}_{n-d+1}, \ldots, \bar{x}_{n}$ is also a s.o.p. of $R / I$. Hence by Lemma 3 the images of the ideal $J=\left(x_{n-d+1}, \ldots, x_{n}\right)$ in $R / I$ and $R / i n(I)$ are a minimal reduction of their maximal homogeneous ideals respectively. By abuse of notation we also denote these images by $J$. We have

$$
\begin{aligned}
& r(R / I) \leq r_{J}(\mathfrak{m})=a\left(R /\left(I, x_{n-d+1}, \ldots, x_{n}\right)\right) \quad(\text { by Lemma } 3) \\
& \quad=a\left(R / i n\left(I, x_{n-d+1}, \ldots, x_{n}\right)\right) \quad(\text { by Macaulay's theorem, see [4], Theorem 15.3) } \\
& \quad \leq a\left(R /\left(i n(I), x_{n-d+1}, \ldots, x_{n}\right)\right) \\
& \quad=r_{J}(R / i n(I)) \quad(\text { by Lemma } 3) \\
& \quad=r(R / \operatorname{in}(I)) \quad(\text { by Theorem } 11) .
\end{aligned}
$$

By changing the indexes of variables, we may assume that $x_{1}>x_{2}>\cdots>x_{n}$. Then we know by Galligo's theorem that there is a Zariski open subset $\mathcal{U} \subseteq G L(n, k)$ such that for each $g \in \mathcal{U}$, $i n(g(I))$ is Borel-fixed (see [4], 15.9.2). Since the new coordinates, obtained by a transformation $g \in \mathcal{U}$, are so called generic coordinates, the last statement follows. 
Also in support of the conjecture by Vasconcelos is the following

Proposition 13. Assume that $R / I$ is a Cohen-Macaulay ring. Then

$$
r(R / I) \leq r(R / \operatorname{in}(I)) .
$$

Proof. Since $R / I$ is assumed to be Cohen-Macaulay, it follows from [7], Proposition 3.2 , that $r(R / I)=a\left(H_{\left(x_{1}, \ldots, x_{n}\right)}^{d}(R / I)\right)$ and $r(R / i n(I)) \geq a\left(H_{\left(x_{1}, \ldots, x_{n}\right)}^{d}(R / \operatorname{in}(I))\right)$, where $d=\operatorname{dim}(R / I)$ and $H_{\left(x_{1}, \ldots, x_{n}\right)}^{d}(R / I), H_{\left(x_{1}, \ldots, x_{n}\right)}^{d}(R / i n(I))$ are local cohomology modules. By [5], Theorem $4, \operatorname{Ext}_{R}^{d}(R / I, R)$ is a subfactor of $\operatorname{Ext}_{R}^{d}(R / \operatorname{in}(I), R)$. Hence by local duality we have

$$
r(R / I)=a\left(H_{\left(x_{1}, \ldots, x_{n}\right)}^{d}(R / I)\right) \leq a\left(H_{\left(x_{1}, \ldots, x_{n}\right)}^{d}(R / \operatorname{in}(I))\right) \leq r(R / i n(I)) .
$$

\section{ON THE RELATION BETWEEN THE REDUCTION NUMBER} AND THE MAXIMAL DEGREE

Let $D(I)$ be the maximum degree of the elements in a minimal generating set for $I$. If $R / I$ is Buchsbaum ring, then $r(R / I)=\operatorname{reg}(R / I) \geq D(I)-1$ by [7], Corollary 3.3 and Lemma 3.4 , where

$$
\operatorname{reg}(R / I)=\max \left\{a\left(H_{\mathfrak{m}}^{i}(R / I)\right)+i ; i \leq d\right\}
$$

is the Castelnuovo-Mumford regularity of $R / I$ (see also [8], Proposition 4.14). One may ask if we always have $D(I) \leq r(R / I)+1$ ? (In this connection see also Remark 4.15 and the diagram on pp. 188 in [8].) However this is not the case, as is exemplified in [8], Example 4.2(b), and our Example 7. By Theorem 11 we even have $r(R / I) \leq D(I)+1$ for stable monomial ideals (with the possibility of a strict inequality). In the following examples we show that the inequality $D(I) \leq r(R / I)+$ 1 does not hold for prime ideals and that in even seemingly simple situations, the reduction number depends on the choice of a minimal reduction.

Example 14. Let $\mathfrak{p} \subset R=k\left[x_{0}, \ldots, x_{3}\right]$ be the prime ideal of the monomial curve $\left(t_{0}^{87}, t_{0}^{40} t_{1}^{47}, t_{0}^{2} t_{1}^{85}, t_{1}^{87}\right)$ in $\mathbf{P}_{k}^{3}$. By [1], Example $2, \mathfrak{p}$ is generated by six binomials $x_{1}^{22}-x_{0}^{10} x_{2}^{5} x_{3}^{7}, x_{0}^{4} x_{2}^{6}-x_{1}^{9} x_{3}, x_{0}^{6} x_{3}^{8}-x_{1}^{13} x_{2}, x_{0}^{2} x_{3}^{9}-x_{1}^{4} x_{2}^{7}, x_{0}^{2} x_{2}^{13}-x_{1}^{5} x_{3}^{10}, x_{2}^{20}-x_{1} x_{3}^{19}$. By Lemma 3 we have $r_{\left(x_{0}, x_{3}\right)}(R / \mathfrak{p})=22$. Using the computer system "Macaulay" by D. Bayer and M. Stillman, we get from Lemma 3 that $r_{\left(x_{0}-x_{1}-x_{2}, x_{3}-x_{1}-x_{2}\right)}(R / \mathfrak{p})=$ 17 ; thus $r(R / \mathfrak{p}) \leq 17$, while $D(\mathfrak{p})=22$.

At present it is not clear to us how to calculate in general the reduction number of the coordinate ring of a monomial curve in $\mathbf{P}^{3}$. We close with the computation of the reduction number for a special class of monomial curves in $\mathbf{P}^{3}$.

Example 15. Let $\mathfrak{p}$ be the defining prime ideal of the monomial curve

$$
\left(t_{0}^{b+a}, t_{0}^{b} t_{1}^{a}, t_{0}^{a} t_{1}^{b}, t_{1}^{a+b}\right)
$$

with g.c.d. $(a, b)=1$ and $b>a>0$. Then

$$
\begin{aligned}
\mathfrak{p} & =\left(x_{0} x_{3}-x_{1} x_{2}, x_{1}^{b}-x_{0}^{b-a} x_{2}^{a}, x_{1}^{b-1} x_{3}-x_{0}^{b-a-1} x_{2}^{a+1}, \ldots, x_{1}^{a} x_{3}^{b-a}-x_{2}^{b}\right) \subset R \\
& =k\left[x_{0}, \ldots, x_{3}\right] .
\end{aligned}
$$

We claim

$$
r_{J}(R / \mathfrak{p})=r(R / \mathfrak{p})=b-1=\operatorname{reg}(R / \mathfrak{p}) .
$$

We have by Lemma $3, r_{\left(x_{0}, x_{3}\right)}(R / \mathfrak{p})=b-1$. Let $J=\left(\bar{y}_{1}, \bar{y}_{2}\right)$ be a minimal reduction of $\mathfrak{m}$. Since $\bar{x}_{0}^{n} \notin\left(\bar{x}_{1}, \bar{x}_{2}, \bar{x}_{3}\right)$ and $\bar{x}_{3}^{n} \notin\left(\bar{x}_{0}, \bar{x}_{1}, \bar{x}_{2}\right)$ for all $n>0$, one 
may assume that either $y_{1}=x_{0}+a_{11} x_{1}+a_{12} x_{2}, y_{2}=x_{3}+a_{21} x_{1}+a_{22} x_{2}$ or $y_{1}=x_{0}+b_{11} x_{1}+b_{12} x_{2}+b_{13} x_{3}, b_{13} \neq 0, y_{2}=b_{21} x_{1}+b_{22} x_{2}$ such that $\left(b_{11}, b_{21}\right)=$ $(0,1)$ or $\left(b_{12}, b_{22}\right)=(0,1)$. Note that every generator in $\mathfrak{p}$ except one has degree $b$. From this it follows that either $x_{1}^{b-1}$ or $x_{2}^{b-1}$ is not in $\left(\mathfrak{p}, y_{1}, y_{2}\right)$. Hence, by Lemma $3, r_{J}(R / \mathfrak{p}) \geq b-1$. Thus $r(R / \mathfrak{p})=b-1$. Moreover, by [2], Theorem 3.1, $\operatorname{reg}(R / \mathfrak{p})=b-1$. Since $r_{J}(R / \mathfrak{p}) \leq \operatorname{reg}(R / \mathfrak{p})$ by [7], Proposition $3.2, r_{J}(R / \mathfrak{p})=b-1$.

\section{ACKNOWLEDGMENT}

This paper was written while both authors were visiting Massey University, New Zealand. For support and the congenial atmosphere in the Department of Mathematics we express our thanks.

\section{REFERENCES}

[1] Bresinsky, H.: Minimal free resolutions of monomial curves in $\mathbf{P}_{k}^{3}$, Linear Alg. Appl. 59(1984), 121-129. MR 85d:14042

[2] Bresinsky, H., F. Curtis, M. Fiorentini, L. T. Hoa: On the structure of local cohomology modules for monomial curves in $\mathbf{P}_{k}^{3}$, Nagoya Math. J. 136(1994), 81-114. MR 96b:14040

[3] Bayer D., M. Stillman: A criterion for detecting $m$-regularity, Invent. Math. 87 (1987), 1-11. MR 87k:13019

[4] Eisenbud, D.: Commutative Algebra with a view towards Algebraic geometry. Springer-Verlag, Berlin-Heidelberg-New York, 1995.

[5] Gräbe, H. J.: Homology modules and standard bases, Beitr. Alg. Geom. 32(1991), 11-20. MR 93c: 13016

[6] Northcott, D. G., D. Rees: Reductions of ideals in local rings, Proc. Camb. Phil. Soc. 50(1954), 145-158. MR 15:596a

[7] Trung, N. V.: Reduction exponent and degree bound for the defining equations of graded rings, Proc. Amer. Math. Soc. 102(1987), 229-236

[8] Vasconcelos V. W.: The degrees of graded modules, In: Proceedings of Summer School on Commutative Algebra, Bellaterra 1996, Vol. II, CRM Publication, 141-196.

Department of Mathematics, University of Maine, Orono, Maine 04469-5752

E-mail address: Henrik@maine.maine.edu

Institute of Mathematics, Box 631, Bò Hô, Hanoi, Vietnam 